\title{
Hidden Markov Based A Target Trajectory Tracing Algorithm
}

\author{
Ding-Jiu YU \\ National Digital Switching System Engineering and Technological Research and Development \\ Center, Zhengzhou Henan, China \\ yudingjiu@163.com
}

Keywords: Target Trajectory Tracking, Hidden Markov Model, Viterbi.

\begin{abstract}
With static position feature of target, existing positioning algorithms can not track target trajectory well when the target is mobile. To solve this problem, a target trajectory tracking algorithm based on hidden Markov model is proposed in this paper. Firstly, a hidden Markov model is established according to the location information within cell coverage area and moving speed of the target. Then, the optimal path and the optimal state probability are solved according to Viterbi decoding algorithm, which realizes the final tracking target trajectory. Simulation results show that this algorithm can obtain accurate target trajectory on the resolution of base station cell.
\end{abstract}

\section{Introduction}

Since the positioning demand of E-911 appeared, the positioning technology in cellular mobile communication systems has been concerned largely. The rapid development of mobile communication technology and the exponential growth of users have promoted the further study of the cellular network positioning technology.

Wireless position technology based on signal feature detection is dependent on wireless environment seriously. In order to obtain a more precise result of location estimation, the line-of-sight (LOS) propagation between transmitter and receiver is needed as well as participation of multiple control-platforms with strict time synchronization [1]. The domestic and foreign scholars have proposed many effective methods to inhibit or reduce NLOS impact to improve the positioning accuracy effectively [2]-[5]. However, each method has its suitable occasions, assumptions and high computational complexity, making it difficult to be applied to the actual positioning system. Especially in complex electromagnetic environment, traditional radio wave propagation models and NLOS distribution model. While, positioning schemes based on the database location information matching show its potential advantages [6][7]. In [8]-[10], H K Lee proposes a positioning method for reducing the NLOS effect to the measured data by establishing the NLOS error database to reduce the NLOS impact to the measured data. In [13] a brief summary to database positioning method is given and a variety of correlation matching methods is presented. In [11] a way of building fingerprint database by training the neural network is introduced. Database positioning error sources, the pros and cons of the deterministic methods and probabilistic methods are analysed in [12]. However, all the existing database positioning algorithms assume the users to be stationary, and then get the positioning results by comparing the user's continuous measured data to the stored prior collected data. But when the target moves, the actual collected data will change constantly, which has a negative impact to the exact match. Meanwhile, the target location information is isolated, just presenting its static position feature. When the target is mobile, the target trajectory can not be well tracked and acquired. Because of the complex actual street, even different targets with the same location information have different trajectories and so on.

To solve the above problem, we design a target trajectory tracking algorithm based on hidden Markov model. Firstly, based on the location information within the cell coverage area(including roads, the situation of segmentation and base station location) and the target's moving speed, it constructs an actual road topology map for target trajectory restore problem, and calculates the transfer matrix between each road. Then, after establishing the hidden Markov model, we use the Viterbi decoding algorithm to solve the optimal path and the optimal state probability, realizing 
tracking and recovering the final target trajectory. Simulation results show that this algorithm can accurately obtain the target trajectory on the base station cell resolution.

\section{System Model}

The model of tracking the target trajectory in accordance with the host cell range is shown in Fig. 1 , in which there are a number of roads within the cell coverage. The road is separated in accordance with the case of the cell coverage, for example, the upper part of the longitudinal road is covered completely by the base station $\mathrm{C}$, while three base stations cover the lower half. Then, the longitudinal road is marked as "1" and "2 "in two parts.

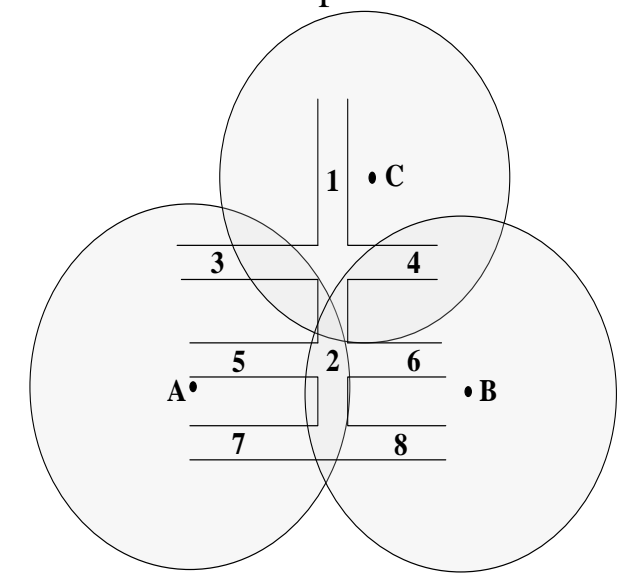

Fig. 1 target trajectory tracking model

Using Hidden Markov model [13] to describe Fig. 1, road segments constitute a states set which contains $\mathrm{N}$ elements. And the set is denoted as

$$
S=\left\{s_{1}, s_{2}, \ldots, s_{N}\right\}
$$

The observation set is composed of base station and moving speed. The collection is recorded as

$$
\begin{aligned}
& Y=\left\{o, v \mid o_{t_{i}} \in\left\{o_{1}, o_{2}, \ldots, o_{M}\right\}\right. \\
& \left., v_{\min } \leq v \leq v_{\text {max }}\right\}
\end{aligned}
$$

Where $\left\{o_{1}, o_{2}, \ldots, o_{M}\right\}$ is the $\mathrm{M}$ base stations, with a moving speed between the $\operatorname{interval}\left(v_{\min }, v_{\max }\right)$. The transition probability from state ${ }^{s_{i}}$ to state ${ }^{s_{j}}$ can be depicted as

$$
\begin{aligned}
& P\left(s_{j} \mid s_{i}\right)=a_{i j}, \\
& i=1,2, \ldots, N, j=1,2, \ldots, N
\end{aligned}
$$

In the state $s_{i}$, the conditional probability density function of observing $\{o, v\}$ can be written as

$$
\begin{aligned}
& P\left(o_{k}, v \mid s_{i}\right)=b_{i}\left(o_{k}, v\right), \\
& k=1,2, \ldots, M, v_{\text {min }} \leq v \leq v_{\max }
\end{aligned}
$$

Therefore, the target trajectory tracking problems is becoming solving the state sequence in the case of knowing observation probability $b_{i}\left(o_{k}, v\right)$, the transition probabilities ${ }^{a_{i j}}$ and observation $\operatorname{set}\left\{y_{t_{1}}, y_{t_{2}}, \ldots, y_{t_{T}}\right\}, y_{t_{i}} \in Y$. 


\section{Tracking Algorithm based on Hidden Markov Model}

Tracking algorithm based on hidden Markov model can be achieved in three steps

1) constructing the topology map;

2) establishing hidden Markov model;

3) calculating optimal solution.

First, according to the location information within the cell coverage area (including roads, the situation of segmentation and base station location) and the moving speed, it constructs the actual road topology map and determines the initial state distribution, the state transition probability distribution matrix and observation process probability density vector. After establishing the hidden Markov model, the target trajectory tracking can be realised based on Viterbi decoding algorithm.

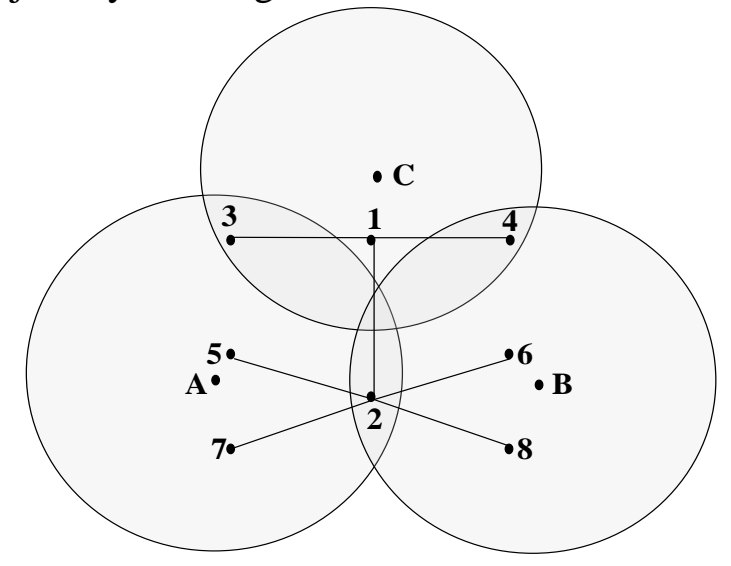

Fig.2 The graph structure of sections as nodes

\section{Construction of Topology Map}

The first step is the preparatory one, mainly constructing topology map of the actual road and calculating the transfer matrix between each two sections of highway.

\section{Construct the Topology Map}

Firstly, the highway distribution map within the base station coverage shown in Fig. 1 is changed into the graph structure shown in Fig. 2, wherein each section of road is represented by a node. As shown in Fig. 2, eight nodes in the coverage of three base station are expressed by eight states $s_{i}(i=1,2, \ldots, 8)$ representing 8 highway sections in Fig. 1.

\section{Set the Transition Probability Matrix}

Transition probability exists in each two of these eight points. As shown in Fig. 2, transition probability between two loads with only one link is $P\left(s_{1} \mid s_{3}\right)=1$. The transition probabilities of the loads with a number of links can be set according to the actual situation. For example, assuming that the 2nd section can only reach No. 6 and No. 8 section with the same traffic flow, we can set $P\left(s_{8} \mid s_{2}\right)=P\left(s_{6} \mid s_{2}\right)=1 / 2$. The transition probability matrix between all eight nodes can be set in the same way.

\section{Set the Conditions for the Observation Probability Matrix}

In practical applications, two kinds of information can be observed, which are the current target moving speed (represented by $v$ ) and the current target cell belonging ((represented by $o$ ). Thus, when the target is in a certain section (in state $s_{i}$ ), there is a certain probability to observe the target speed and its cell property. For example, when the target is in the section 2, it may be presented in the cell A or cell B so that $P\left(o_{A} \mid s_{2}\right)=P\left(o_{B} \mid s_{2}\right)=1 / 2$. In addition, when the target is in section 2 with the speed of $v$, the conditional probability density function can be defined as $f\left(v \mid s_{2}\right)$. Therefore, according to the actual situation the conditional probability distribution matrix of these two 
observations can be pre-built.

\section{Establishing Hidden Markov Model}

The second step is to set a hidden Markov model. As shown in Fig. 3, when the position and speed of the base station are successively observed as $\left\{A, v_{1}\right\},\left\{B, v_{2}\right\},\left\{C, v_{3}\right\}$ and $\left\{A, v_{4}\right\}$, the optimal path probability $\phi_{s_{j}}(t+1)$ and the optimal state probability $\psi_{s_{j}}(t+1)$ in Fig. 3 can be calculated in accordance with (6) and (7).

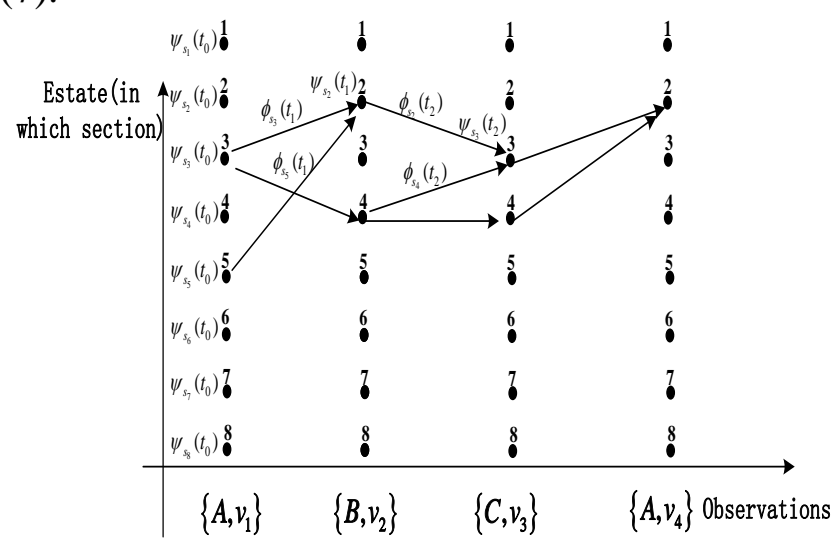

Fig.3 The graph structure of sections as nodes

\section{Calculating Optimal Solution}

The third step is to use Viterbi algorithm to calculate the optimal solutions. When completing the probability calculation in accordance with Fig. 3, we can take advantage of Viterbi decoding to find out the section (state) that includes the target [13][14].

Assume that the probability distribution of the street including the target from the initial time to its initial state distribution, recorded as $\pi_{n}, n=1,2, \ldots, N$, and $\sum_{n=1}^{N} \pi_{n}=1$. After $\mathrm{T}$ times observations $\left\{y_{t_{1}}, y_{t_{2}}, \ldots, y_{t_{T}}\right\}, s_{i}$ is the state at the moment $t_{T}$ and the optimal path probability of being in the state $s_{i}$ at the moment $t$ is $\phi_{s_{i}}(t), i=1,2, \ldots, N$. The observation value set observed at the moment $t_{T+1}$ is $y_{t_{T+1}}$, and the probability in the state $t_{T+1}$ can be written as

$$
\begin{gathered}
P\left(x_{t_{T}}=s_{i}, y_{t_{1}}, y_{t_{2}}, \ldots, y_{t_{T}}, y_{t_{T+1}}, x_{t_{T+1}}=j\right) \\
=P\left(x_{t_{T}}=s_{i}, y_{t_{1}}, y_{t_{2}}, \ldots, y_{t_{T}}\right) . \\
P\left(x_{t_{T+1}}=s_{j} \mid x_{t_{T}}=s_{i}\right) \cdot P\left(y_{t_{T+1}} \mid x_{t_{T+1}}=s_{j}\right) \\
=\phi_{s_{i}}(t) \cdot a_{i j} \cdot b_{j}\left(y_{t_{T+1}}\right)
\end{gathered}
$$

Therefore, at the moment $t_{T+1}$, the optimal path probability of the target being in state $s_{j}$ can be shown as

$$
\phi_{s_{j}}(t+1)=\max _{S, v_{\min } \leq v \leq v_{\max }}\left(\phi_{s_{i}}(t) \cdot a_{i j}\right) \cdot b_{j}\left(y_{t_{r+1}}\right)
$$

From $t_{T}$ to $t_{T+1}$, the target is denoted as

$$
\psi_{s_{j}}(t+1)=\underset{s_{i} \in S}{\arg \max }\left(\phi_{s_{i}}(t) \cdot a_{i j}\right)
$$

According to Viterbi algorithm based on (6) and (7), we can obtain the most likely position of 
target from the moment $t_{T}$ to moment $t_{T}$ as well as the target trajectory by connecting these positions together.

\section{Performance Simulation}

\section{Trajectory Tracking Performance}

As is shown in Fig. 4, there are 25 samples of the target trajectory in the 8 streets, the observation values in each sample are shown in Table 1.

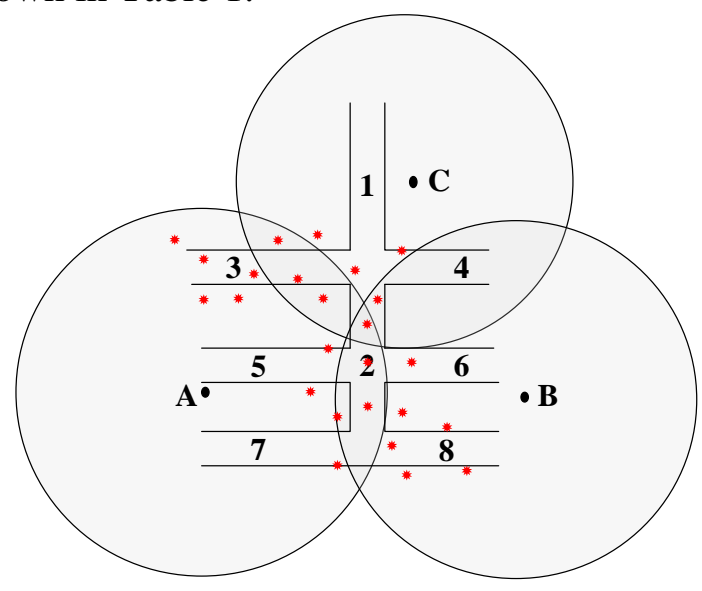

Fig.4 Sample distribution of sections simulation.

Tab.1 The observation sample data

\begin{tabular}{|c|c|c|c|c|c|c|}
\hline sample point number & 1 & 2 & 3 & 4 & 5 & 6 \\
\hline BS number & A & A & A & C & A & C \\
\hline Speed km/h & 85 & 77 & 79 & 83 & 88 & 80 \\
\hline sample point number & 7 & 8 & 9 & 10 & 11 & 12 \\
\hline BS & A & A & C & C & C & B \\
\hline Speed km/h & 78 & 79 & 60 & 62 & 74 & 79 \\
\hline sample point number & 13 & 14 & 15 & 16 & 17 & 18 \\
\hline BS & B & C & A & B & C & A \\
\hline Speed km/h & 87 & 81 & 85 & 79 & 77 & 78 \\
\hline sample point number & 19 & 20 & 21 & 22 & 23 & 24 \\
\hline BS & A & A & B & B & B & B \\
\hline Speed km/h & 68 & 66 & 61 & 70 & 73 & 78 \\
\hline sample point number & 25 & & & & & \\
\hline BS & B & & & & & \\
\hline Speed km/h & 84 & & & & & \\
\hline
\end{tabular}

Create a hidden Markov model for this problem, and use Viterbi algorithm based on (6) and (7) to solve the solution. The specific processes as follow

1 Initialization:

$1.1 \delta_{1}(i)=p_{i} b_{i, o_{1}}, i=1,2, \cdots, 8$;

$1.2 \psi_{1}(i)=0 ; i=1,2, \cdots, 8$;

2 Recursive calculation:

$2.1 \delta_{t}(j)=\left[\max _{i=1,2, \cdots, 8}\left(\delta_{t-1}(i) a_{i, j}\right)\right] b_{j, o_{t}}, j=1,2, \cdots, 8, t=2, \cdots, 25$;

$2.2 \psi_{t}(j)=\arg \max _{i=1,2, \cdots, 8}\left[\delta_{t-1}(i) a_{i, j}\right], j=1,2, \cdots, 8, t=2, \cdots, 25$;

3 Terminal calculation:

$3.1 P^{*}=\max _{i=1,2, \cdots, 8}\left[\delta_{T}(i) q_{i}\right]$;

$3.2 z_{T}^{*}=\arg \max _{i=1,2, \cdots, 8}\left[\delta_{T}(i) q_{i}\right]$; 
4 Backtracking the optimal path:

$z_{t}^{*}=\psi_{t+1}\left(z_{t+1}^{*}\right), t=24, \cdots, 1$.

We will obtain the sample classification shown in Fig. 5. As is shown in Table 2

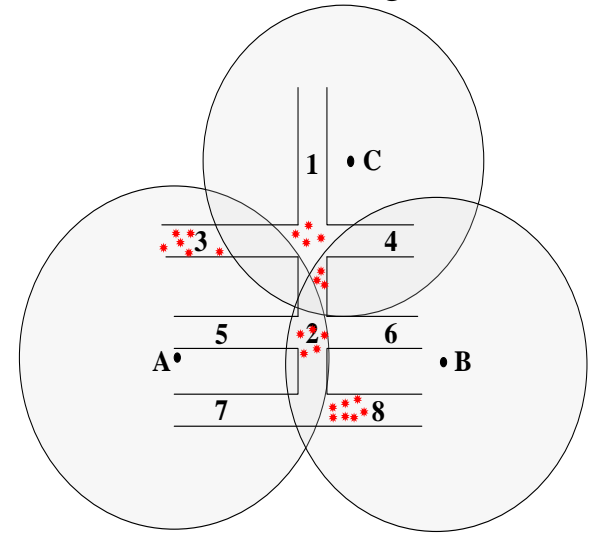

Fig.5 Sample distribution after Viterbi decoding

Tab.2 Classification of Viterbi algorithm

\begin{tabular}{|c|c|}
\hline $\begin{array}{c}\text { Steet } \\
\text { number }\end{array}$ & Sample point number \\
\hline Steet 1 & $7,8,9$ \\
\hline Steet 2 & $13,14,15,16$ \\
\hline Steet 3 & $1,2,3,4,5,6$ \\
\hline Steet 4 & $10,11,12$ \\
\hline Steet 5 & 17, \\
\hline Steet 6 & 18, \\
\hline Steet 7 & 19,20, \\
\hline Steet 8 & $21,22,23,24,25$ \\
\hline
\end{tabular}

The hidden Markov models can be used to track target trajectory. The accuracy will continue to increase with the increasing number of the observation points. And the more precisely the block divided, the better tracking precision will be obtained.

\section{The Error Performance of Trajectory Tracking}

In addition, the observation information (cell belonging and moving speed) may be error, leading to the trajectory tracking results error. Therefore, we need to analyze the wrong case of trajectory tracking when the observation error exists. Assuming that there are 10 cells, the simulation results of each cell belonging and speed is shown in Fig. 6. Wherein the abscissa is the observation information error rate and ordinate is the trajectory tracking error rate (the error probability of the tracking state). The solid line is the simulation result while the dashed line is the fitting result. Fitting is:

$$
y=\frac{1+\mathrm{e}^{-\left(\mathrm{B}_{0}+\mathrm{B}_{1}\right)}}{1+\mathrm{e}^{-\left(\mathrm{B}_{0}+\mathrm{B}_{1} \mathrm{x}\right)}}
$$

Wherein, $\mathrm{y}$ is error probability of trajectory tracking and $\mathrm{x}$ is the error probability of observation information (speed or cell belonging information). B0, B1 are the fitting parameters.

In Fig. 6, if there is an error in the observed speed while the cell belonging is correct, fitting parameters is $\mathrm{B} 0=-6.33, \mathrm{~B} 1=10.03$; if there is an error in the cell belonging while the observed speed is correct, fitting parameters is $\mathrm{B} 0=-4.72$, B1 $=9.07$. According to Fig. 6 , the distribution trends in the two error cases are similar, however the impact of the speed error is less.

Fig. 7 shows the error probability curve of the trajectory according to the 20 sequences in the 20 
cells, wherein each cell is observed once. In Fig. 7, if there is an error in the observed speed while the cell belonging is correct, the fitting parameters is $\mathrm{B} 0=-6.21, \mathrm{~B} 1=9.98$; if there is an error in the cell belonging while the observed speed is correct, fitting parameters is $\mathrm{B} 0=-4.66, \mathrm{~B} 1=8.93$. Comparing to Fig. 6, the improvement of increasing the observation sequence length, for the trajectory tracking results is not large.

Fig. 8 shows the error probability curve of the trajectory according to the 20 sequences in the 10 cells, wherein each cell is observed twice. In Fig. 8, if there is an error in the observed speed while the cell belonging is correct, the fitting parameters are $\mathrm{B} 0=-9.33, \mathrm{~B} 1=12.03$; if there is an error in the cell belonging while the observed speed is correct, fitting parameters are $\mathrm{B} 0=-7.73, \mathrm{~B} 1=11.02$. Comparing Fig. 8 and Fig. 7, increasing the number of observation points within the cell improves trajectory tracking results.

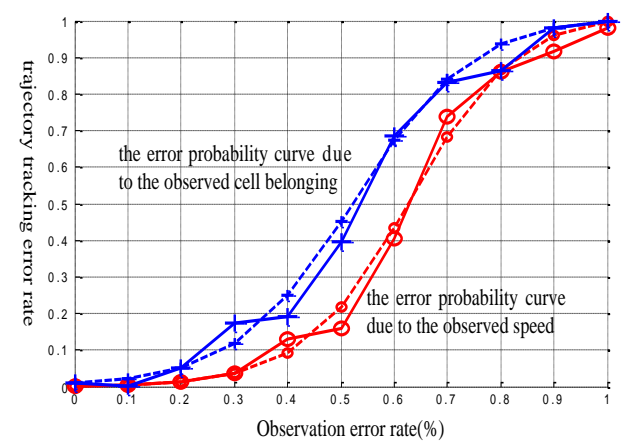

Fig. 6 The error probability curve of single observation point in 10 cells

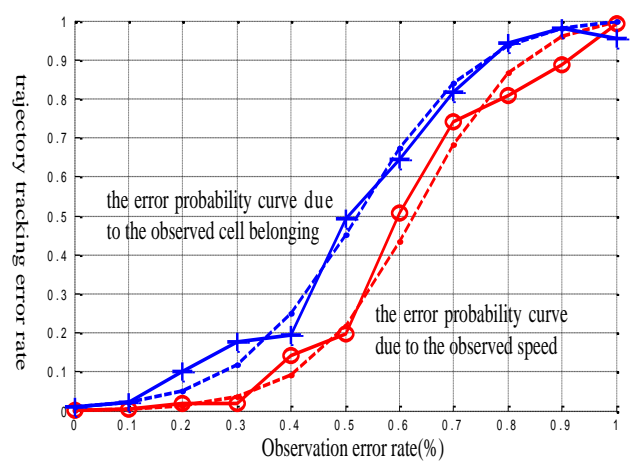

Fig.7 The error probability curve of single observation point in 20 cells

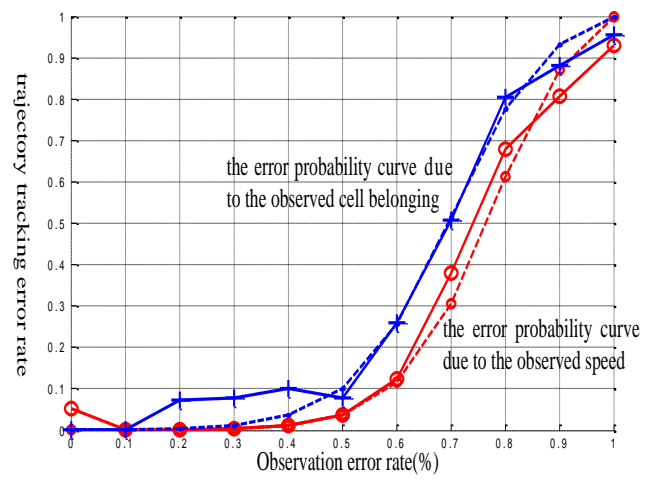

Fig. 8 The error probability curve of two observation points in 20 cells

Fig. 9 shows the error probability curve of the trajectory according to the 100 sequences in the 10 cells, wherein each cell is observed 10 times. In Fig. 9, if there is an error in the observed speed while the cell belonging is correct, the fitting parameters are $\mathrm{B} 0=-9.73, \mathrm{~B} 1=12.68$; if there is an 
error in the cell belonging while the observed speed is correct, fitting parameters are $\mathrm{B} 0=-8.03, \mathrm{~B} 1$ $=$ 11.75. Comparing Fig. 8 and Fig. 9, the correct rate of trajectory tracking can be improved steadily with the increasing number of the observation points, but the degree of increasing becomes gradually smaller.

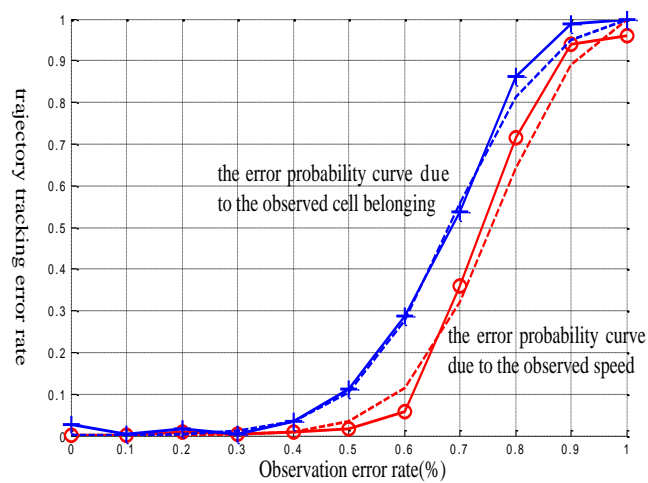

Fig.9 The error probability curve of multiple observation points in 10 cells

\section{Conclusion}

In this paper, a target trajectory tracking algorithm based on hidden Markov model is proposed for mobile target tracking problem. Firstly, a hidden Markov model is established according to the location information within cell coverage area and moving speed of the target. Then, the optimal path and the optimal state probability are solved according to Viterbi decoding algorithm, which realizes tracking final target trajectory. The simulation results show that this algorithm can obtain accurate target trajectory on the resolution of base station cell.

\section{References}

[1] J. Caffery. Wireless Location in CDMA Cellular Radio Systems [M]. Norwell: Kluwer Academic Publishers, 2001.

[2] J. Borras, P.Hatrack, N.B. Mandayam. Decision Theoretic Framework for NLOS Identification [A]. In: Proc. of IEEE Vehicular Technology Conference (VTC)[C], 1998, 2:1583-1587.

[3] C. Ma, Richard Klukas, Gérard Lachapelle. Nonline -of- Sight Error- Mitigation Method for TOA Measurements [J]. IEEE Transactions on Vehicular Technology, 2007, 56(2):641-651.

[4] C. Botteron,A.Host-Madsen,M.Fattouche. Cramer-Rao Bounds for the Estimation of Multipath Parameters and Mobiles Positions in Asynchronous DS-CDMA Systems[J]. IEEE Transactions on Signal Processing,2004,52(4): 862-875.

[5] Y. Qi,H.Kobayashi,H.Suda. Analysis of Wireless Relocation in a Non-Line-of-Sight Environment[J]. IEEE Transactions on Wireless Communications,2006,5(3): 672-681.

[6] H. Laitinen, T. Nordstrom, J. Lahteenmaki. Database Correlation Method for GSM Location [A]. In: Proc. of IEEE Vehicular Technology Conference (VTC)[C], Rhodes, Greece, May 2001.

[7] D. Zimmermann, J. Baumann, M. Layh, et al. Database Correlation for Positioning of Mobile Terminals in Cellular Networks using Wave Propagation Models [J]. 2004: 4682-4686.

[8] B. Li, Y. Wang, H.K. Lee, A. Dempster, C. Rizos. Method for yielding a database of location fingerprints in WLAN [A]. In: Proc. of IEEE Communications[C], Oct. 2005, 152(5): 580-586.

[9] B. Li, C. Rizos, H.K. Lee. Utilizing kriging to generate a NLOS error correction map for network based mobile positioning [A]. In: Proc. of 2004 International Symposium on GPS/GNSS[C], Sydney, Australia, Dec. 2004: 179. 
[10]H. K. Lee, B. Li, C. Rizos. Implementation procedure of wireless signal map matching for location-based services [A]. In: Proc. of 2005 IEEE International Symposium on Signal Processing and Information Technology (ISSPIT)[C], Athens, Greece, Dec. 2005: 429-434.

[11]W. Tang, Shan Li, Wangqiu Kuang. New algorithm based on spatial correlation for yielding fingerprints data of RF indoor localization. Computer Engineering and Applications, 2008, 44(23): 226-229.

[12]S. Wang. Research on Fingerprint Based WLAN Indoor Location Method[D]. Harbin: Journal of Harbin Institute of Technology, Jun, 2009.

[13]D. Lu. Random process and its application[M]. Tsinghua University Press. 1986.

[14]J. Zhang. Linear model parameter estimation and improved[M]. National University of Defense Technology Press, 1999. 